\title{
Successful prevention of tumor lysis syndrome using recombinant urate oxidase in patient with metastasic and bulky prostate rhabdomyosarcoma
}

\author{
Atsuko Watanabe ${ }^{1,2^{*}}$, Ryuhei Tanaka ${ }^{2}$ \\ ${ }^{1}$ Department of Pediatrics, Kasumigaura Medical Center, Tsuchiura, Japan; *Corresponding Author: atsuko-w@gf7.so-net.ne.jp \\ ${ }^{2}$ Department of Pediatric Oncology/Hematology, International Medical Center, Saitama Medical University, Hidaka, Japan
}

Received 27 November 2013; revised 20 December 2013; accepted 5 January 2014

Copyright (C) 2014 Atsuko Watanabe, Ryuhei Tanaka. This is an open access article distributed under the Creative Commons Attribution License, which permits unrestricted use, distribution, and reproduction in any medium, provided the original work is properly cited. In accordance of the Creative Commons Attribution License all Copyrights (C) 2014 are reserved for SCIRP and the owner of the intellectual property Atsuko Watanabe, Ryuhei Tanaka. All Copyright (C) 2014 are guarded by law and by SCIRP as a guardian.

\section{ABSTRACT}

Tumor lysis syndrome (TLS) is a life-threatening oncological emergency that frequently occurs in patients with hematological malignancies. It is becoming more common in patients with solid tumors because of advances in molecular targeted therapies. Recombinant urate oxidase (rUO) is effective at preventing and treating hyperuricemia, but clinicians who treat adult patients with solid tumors are generally not aware of this. In addition, the treatment guidelines for TLS do not include indications for rUO treatment for chemosensitive sarcoma. We report an adolescent case of metastatic rhabdomyosarcoma (RMS), in which clinical TLS was successfully prevented using rUO. A 16-year-old Japanese male suffered from urinary retention and bone pain and was diagnosed with prostate RMS combined with multiple bone metastases and bone marrow involvement. He was judged to be at high risk of clinical TLS because his prostate tumor was bulky and he displayed laboratory TLS. rUO was administered during chemotherapy. Soon after the initiation of chemotherapy, his disseminated intravascular coagulation (DIC) got worse, and his lactate dehydrogenase (LDH) level was elevated due to tumor lysis. However, his serum uric acid levels remained low, and he was prevented from falling into acute renal failure. The planned regimen was successfully completed without life-threatening complications, and the patient achieved a complete response after 2 courses of chemotherapy. The international TLS consensus panel de- veloped recommendations for TLS prophylaxis, but did not define the TLS risk classification of RMS. We recommend that RMS should be treated like neuroblastoma because it grows rapidly and is highly chemosensitive. Our patient was considered to be indicated for rUO because he displayed urinary retention, DIC, and laboratory TLS before chemotherapy. These features might be useful as indications for rUo therapy, which can safely support chemotherapy.

\section{KEYWORDS}

Tumor Lysis Syndrome; Recombinant Urate Oxidase; Rhabdomyosarcoma

\section{INTRODUCTION}

Tumor lysis syndrome (TLS) is a life-threatening oncological emergency that results from the rapid destruction of malignant cells. The resultant release of intracellular metabolites can lead to hyperuricemia, hyperphosphatemia, hyperkalemia, and/or hypocalcemia. These complications predispose the patient to clinical toxicities including acute renal failure (ARF), cardiac arrhythmia, seizures, and sudden death. TLS is most commonly encountered in chemosensitive hematological neoplasms such as Burkitt's lymphoma and acute lymphoblastic leukemia, but is rarely seen in solid tumors.

The standard management strategy for hyperuricemia consists of hydration, alkalization of the urine, and the oral administration of allopurinol. Allopurinol inhibits xanthine oxidase, which prevents the production of further uric acid (Figure 1). However, there are several pro- 


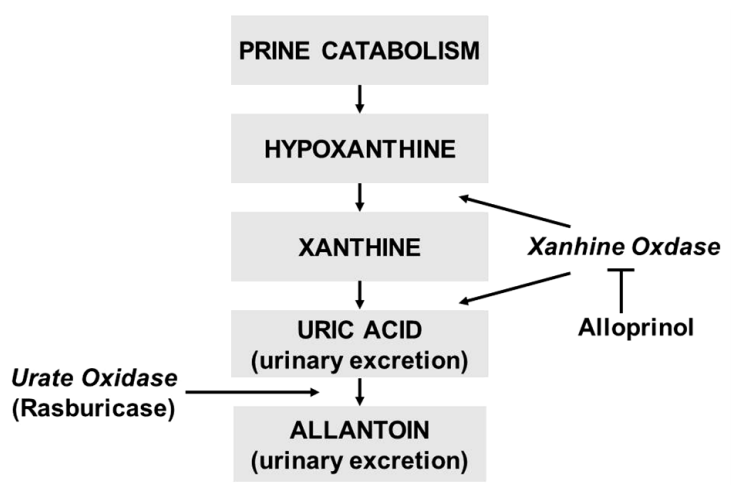

Figure 1. Purine catabolism (From reference No. 1).

blems with this method; i.e., oral allopurinol is difficult to keep compliance for infants and patients with poor general conditions, and it has no direct effect on existing uric acid. The maximal solubility of uric acid occurs at $\mathrm{pH}$ 7.5; on the other hand, the solubility of xanthine and hypoxanthine decreases in alkali condition, which can lead to the production of xanthine crystals.

Recombinant urate oxidase (rUO) converts uric acid into the soluble compound allantoin, which is 5 to 10 times more soluble than uric acid in urine. As a result, it rapidly reduces uric acid levels, and therefore, does not induce the accumulation of xanthine and hypoxanthine. In a randomized trial involving pediatric leukemia and lymphoma patients, rUO was more effective and fast-acting than allopurinol at controlling uric acid [1]. Furthermore, rUO is an intravenous medicine and can be administrated more precisely.

rUO has now been approved in Japan for the initial management of serum uric acid levels in both adult and pediatric patients who are receiving anti-cancer therapies that are expected to cause TLS. It is frequently used for hematological malignancies and pediatric patients with chemosensitive and non-epithelial cancers such as neuroblastoma. Patients with these conditions are usually diagnosed at an advanced stage because these tumors grow rapidly and metastasize easily; therefore, they are at high risk of TLS during the initial stages of treatment. On the other hand, the optimal management strategy for TLS in adult patients with epithelial cancer is poorly understood. In recent years, some patients with chemoresistant epithelial cancer have been reported to develop TLS and ARF after using molecular targeted agents [2]. If it is left undiagnosed or diagnosed too late, TLS leads to death in $20 \%-50 \%$ of cases.

We report the case of an adolescent patient with rhabdomyosarcoma (RMS), in whom rUO successfully prevented renal insufficiency.

\section{CASE PRESENTATION}

A 16-year-old Japanese male suffered repeated epi- sodes of urinary retention from August 2010, was diagnosed with a neurogenic bladder, and was instructed to undergo clean intermittent catheterization by a general practitioner. He had a fever, found urination and bowel voiding difficult, and could not walk because of hip joint pain 3 months later. A computed tomography (CT) scan showed that his prostate gland was swollen so he was transferred to our hospital. Magnetic resonance imaging (MRI) demonstrated a swollen prostate and that a soft tissue mass (diameter: $10 \mathrm{~cm}$ ) was compressing the rectum, and detected multiple swollen small pelvic lymph nodes (Figure 2). An 18F-fluorodeoxy glucose positron emission tomography (FDG-PET) scan detected multiple bone metastases (Figure 3), and bone marrow involvement was revealed by a bone marrow biopsy. Cystoscopy

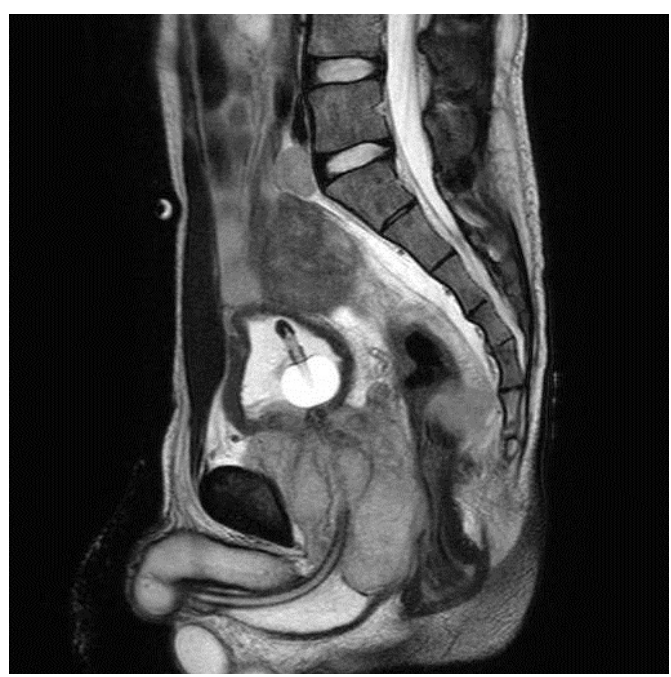

Figure 2. Pelvis MRI at diagnosis.

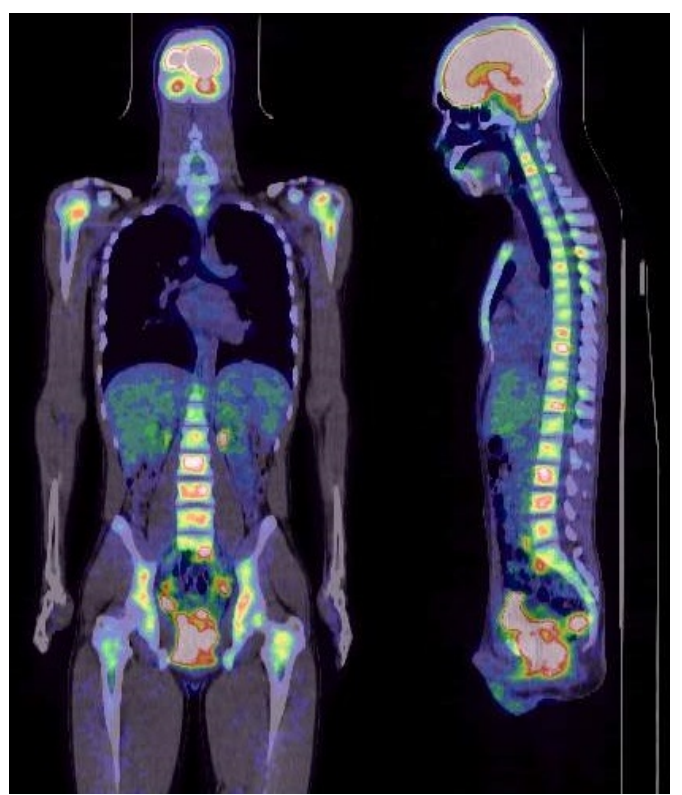

Figure 3. FDG-PET scan at diagnosis. 
and a prostate biopsy were performed, which revealed tumor invasion into the bladder wall. Pathologically, the biopsy specimen contained small round tumor cells (Figure 4(a)), which were found to be positive for desmin and CD56 in an immunohistochemical analysis. Genetic analysis using fluorescence in-situ hybridization (FISH) detected the FKHR split signal (Figure 4(b)), and the tumor cells displayed the $\mathrm{t}(2 ; 13)$ (q36; q14) karyotype. The final diagnosis was metastatic alveolar RMS.

Although the patient's serum uric acid and phosphate levels were elevated (UA: $8.3 \mathrm{mg} / \mathrm{dl}, \mathrm{P}: 6.0 \mathrm{mg} / \mathrm{dl}$ ) due to his laboratory TLS, his creatinine, potassium, and calcium levels were within normal limits. Markedly elevated lactate dehydrogenase (LDH) levels and the progression of disseminated intravascular coagulation (DIC) were observed just one week after the biopsy. The patient was judged to be at high risk of clinical TLS, because RMS is a chemosensitive tumor, and he had a bulky tu-

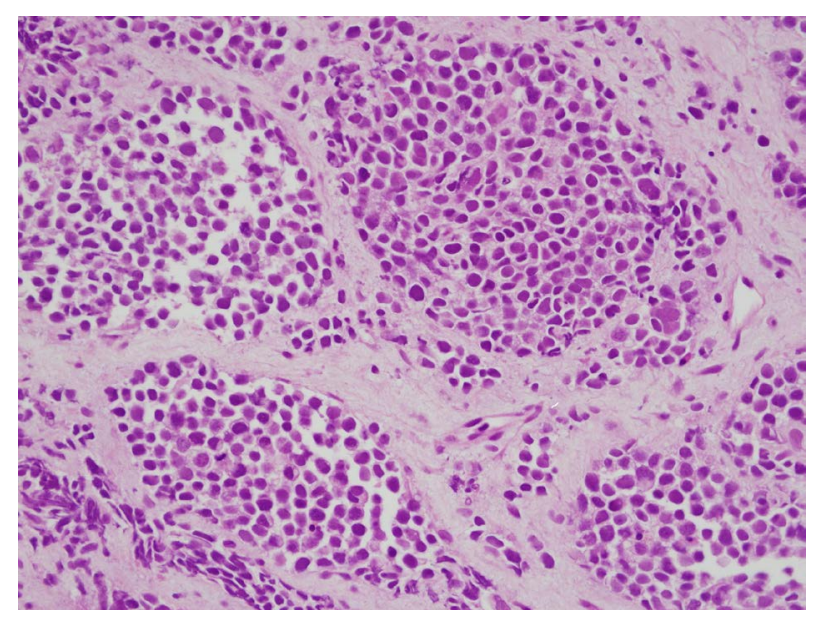

(a)

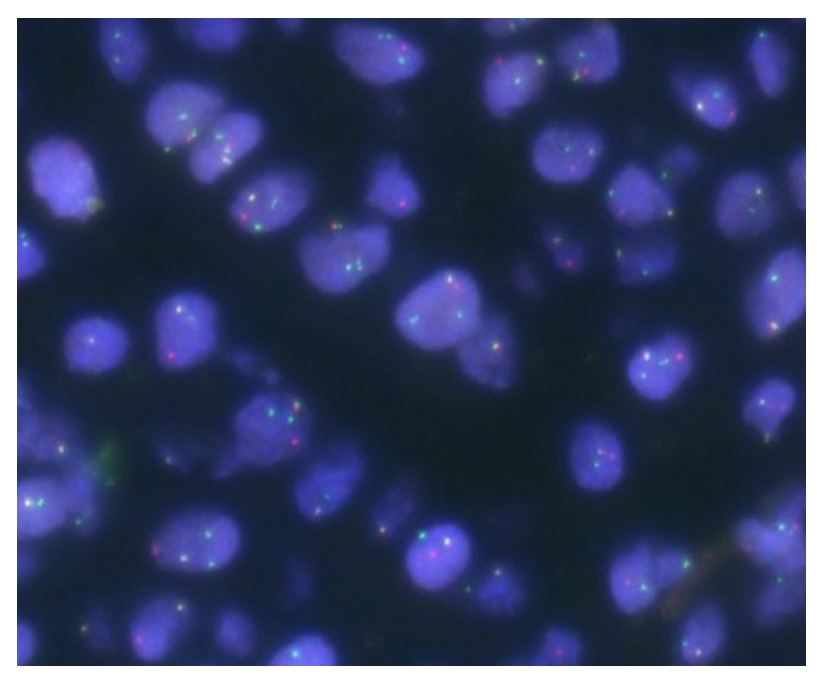

(b)

Figure 4. (a) Histological findings of prostate biopsy, HE stain (×400); (b) FKHR break-apart rearrangement by fluorescence in situ hybridization (FISH). mor and laboratory TLS at diagnosis. rUO was administered at a dose of $7.5 \mathrm{mg} /$ day $(0.14 \mathrm{mg} / \mathrm{kg})$. Although this was less than the recommended dose, we planned to increase the dose according to the grade of the patient's TLS and the effect of the rUO.

During the rUO treatment, we installed a urinary catheter and provided supportive care with narcotic medication to ensure that the anti-cancer therapy was a success.

The VAC regimen $\left(1.5 \mathrm{mg} / \mathrm{m}^{2}\right.$ vincristine (VCR) + $0.045 \mathrm{mg} / \mathrm{kg}$ actinomycin D (ActD) $+2200 \mathrm{mg} / \mathrm{m}^{2}$ cyclophosphamide (CY); all medicines were prescribed on day 1) was employed, as it is the standard chemotherapy for RMS. We administered the VCR on day 1 and the CY on day 2 because we wanted to avoid severe TLS developing and/or the deterioration of the patient's DIC. As predicted, the patient's DIC got worse due to tumor lysis on day 3 , which resulted in the urinary catheter becoming obstructed due to bleeding in the urinary bladder. Consequently, we were forced to perform a cystostomy and administer blood transfusions. As a result of the patient's tumor lysis, his LDH levels rose to 2016 IU/l and his fibrinogen levels decreased to $98 \mathrm{mg} / \mathrm{dl}$, but his serum uric acid levels remained lower than $0.8 \mathrm{mg} / \mathrm{dl}$, and ARF was prevented. We were eventually able to treat him with ActD on day 7 after an improvement in his DIC. He subsequently displayed significant symptomatic improvement, and a CT scan only revealed mild swelling of the prostate. As we considered the VAC regimen to have been effective, we repeated it every 3 weeks. He achieved a complete response after 2 courses of the regimen. Although the patient suffered a grade 4 hematological toxicity and grade 3 febrile neutropenia, he finished all of the scheduled therapy in September 2011.

Unfortunately, recurrence was detected in the pelvic lymph nodes 3 months later. However he underwent salvage chemotherapy, he died two and half years after diagnosis.

\section{DISCUSSION}

TLS is frequently associated with hematological malignancies at the initiation of cytotoxic agent treatment. In general, solid tumors in adults are not sufficiently chemosensitive for chemotherapy to induce TLS. However, the risk of TLS has been overlooked in molecular targeted therapies in recent years. In addition, the utility of rUO for TLS prophylaxis in solid tumor patients is not widely known. In fact, solid tumor patients only account for $1 \%$ of all rUO users. Delays in starting treatment for TLS lead to a poor prognosis, and therefore, early recognition of patients who are at risk of TLS and the initiation of therapy for the condition are essential.

In the Cario-Bishop classification [3], TLS is classified as laboratory or clinical TLS. Laboratory TLS re- 
quires two or more of the following metabolic abnormalities to be present: hyperuricemia, hyperkalemia, hyperphosphatemia, and hypocalcemia. Clinical TLS presents with an increased creatinine level, arrhythmia, seizures, or death.

An international TLS consensus panel developed a risk classification model and prophylaxis recommendations for TLS in 2008 [4]. Most solid tumors are classified as low risk; however, bulky chemosensitive tumors such as small lung cell cancers, germ cell tumors, and neuroblastoma are associated with an intermediate risk of TLS. Furthermore, they are classified as high risk if the patient displays renal dysfunction and/or laboratory TLS, and the use of rUO is recommended for such cases (Figure 5).

Historically, in studies that did not involve rUO 10\% $20 \%$ of patients with hematological malignancies required hemodialysis, but a more recent study found that the use of rUO decreased the frequency of hemodialysis to just a few percent [5].

We surmised that our patient had a large amount of tumor cells in his body due to his bulky prostate mass, multiple bone metastases, and bone marrow involvement. He also suffered from urinary retention and post-renal ARF, which resulted in laboratory TLS; hence, he was classified as being at high risk of clinical TLS and so was administered rUO. He developed severe DIC and tumor bleeding in the bladder soon after the initiation of chemotherapy. However, his serum uric acid levels were successfully controlled with rUO, and he did not develop ARF.

As introducing hemodialysis whilst the patient was suffering from DIC would have been risky, the fact that the rUO treatment had successfully prevented ARF was a great advantage in this case.

Although Cario et al. did not produce a risk classification for RMS, we recommend that it should be treated like neuroblastoma because it grows rapidly and is highly

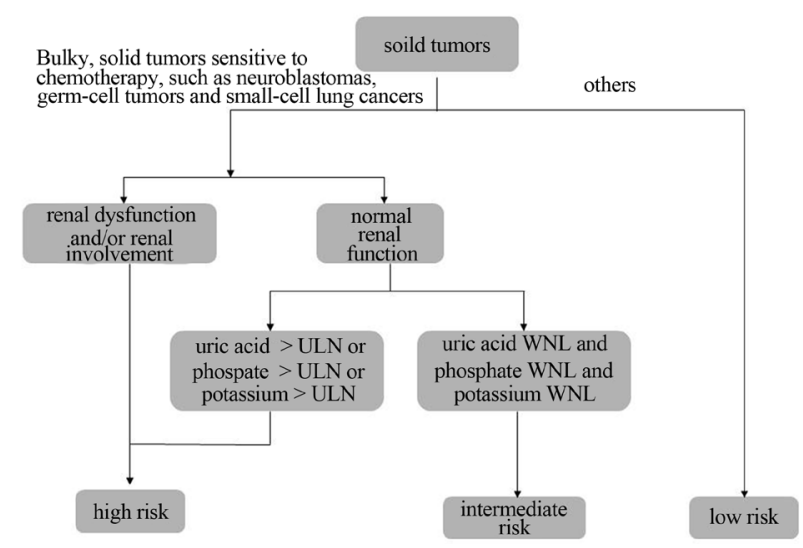

Figure 5. TLS risk assessment of solid tumor (Partially alteration of reference No. 4). chemosensitive.

According to the TLS guidelines, we should pay attention to clinical TLS at the beginning of chemotherapy. In recent years, TLS has been reported in non-small cell lung cancer, breast cancer, renal cell carcinoma, gastrointestinal stromal tumor, etc. All of these cases were treated with molecular targeted agents [2]. Unfortunately, clinicians who treat patients with such tumors are often not aware of the utility of rUO for treating TLS. It is also necessary to re-examine the guidelines for TLS as the therapies for non-epithelial tumors develop.

The recommended dose of $\mathrm{rUO}$ is $0.2 \mathrm{mg} / \mathrm{kg}$ for 3 - 7 days. Limited experience suggests that lower doses of rUO are also effective at lowering uric acid levels [6,7]. When $7.5 \mathrm{mg} / 1 \mathrm{~V}$ of rUO $(0.14 \mathrm{mg} / \mathrm{kg})$ was administered to our patient, his uric acid levels were sufficiently decreased. As rUO is expensive, we suggest that rUO treatment should be started at a low dose and then increased according to the patients' reactions to therapy and the changes in their uric acid levels.

Although the major cause of ARF is uric acid nephropathy secondary to tumor lysis, hyperphosphatemia also induces severe ARF, which can lead to a requirement for hemodialysis [5]. Hyperkalemia, which can result in severe arrhythmia and sudden death, is another potentially a life-threatening consequence of TLS. We should keep in mind that TLS cannot be avoided by controlling the patients' uric acid levels alone.

\section{CONCLUSION}

rUO is a useful option to prevent TLS due to not only hematological malignancies but also solid tumors such as RMS. We also recommend using rUO in a case of pediatric patient because of the better compliance.

\section{REFERENCES}

[1] Goldman, S.C., Holcenberg, J.S., Finklestain, J.Z., Hutchinson, R., Kreissman, S., Johnson, F.L., Tou, C., Harvey, E., Morris, E. and Cario, M. S. (2001) A randomized comparison between rasburicase and allopurinol in children with lymphoma or leukemia at high risk for tumor lysis. Blood, 97, 2998-3003. http://dx.doi.org/10.1182/blood.V97.10.2998

[2] Bose, P. and Qubaiah, O. (2011) A review of tumor lysis syndrome with targeted therapies and the role of rasburicase. Journal of Clinical Pharmacy and Therapeutics, 36, 299-326. http://dx.doi.org/10.1111/j.1365-2710.2011.01260.x

[3] Cario, M.S. and Bishop, M. (2004) Tmor lysis syndrome: New therapeutic strategies and classification. British Journal of Haematology, 127, 3-11. http://dx.doi.org/10.1111/j.1365-2141.2004.05094.x

[4] Cairo, M.S., Coiffier, B., Reiter, A. and Younes, A. (2010) Recommendations for the evaluation of risk and prophy- 
laxis of tumor lysis syndrome (TLS) in adults and children with malignant disease: An expert TLS panel consensus. British Journal of hematology, 149, 578-586.

[5] Jeha, S., Kantarijian, H., Irwin, D., Shen, V., Shenoy, S., Blaney, B., Camitta, B. and Pui, C.H. (2005) Efficacy and safety of rasbricase, a recombinant urate oxidase, in the management of malignancy-associated hyperuricemia in pediatric and adult patients: Final results of a multicenter compassionate use trial. Leukemia, 19, 34-38.

[6] Cammalleri, L. and Malaguarnera, M. (2007) Rasburicase represents a new tool for hyperuricemia in tumor lysis syndrome and in gout. International Journal of Medical sciences, 4, 83-93. http://dx.doi.org/10.7150/ijms.4.83

[7] Trifilio, S., Gordon, L., Singhal, S., Tallman, M., Evans, A., Rashid, K., Fishman, M., Mashio, K., Pi, J. and Mehta, J. (2006) Reduced-dose rasbricase in adult cancer patients with hyperuricemia. Bone Marrow Transplantation, 26, 806-812. 